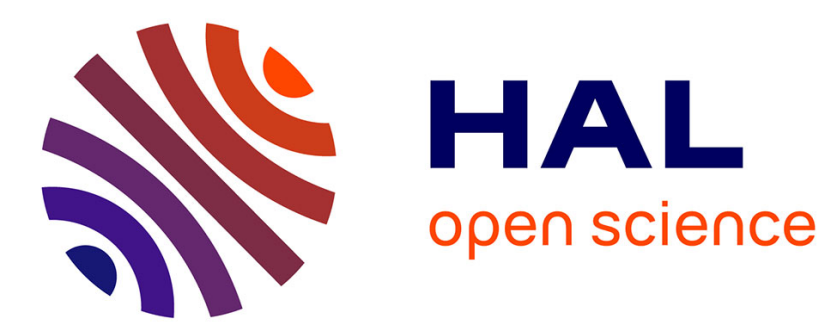

\title{
THE QUADRATIC DYNATOMIC CURVES ARE SMOOTH AND IRREDUCIBLE
}

\author{
Xavier Buff, Lei Tan
}

\section{To cite this version:}

Xavier Buff, Lei Tan. THE QUADRATIC DYNATOMIC CURVES ARE SMOOTH AND IRREDUCIBLE. 2011. hal-00610714

\section{HAL Id: hal-00610714 https://hal.science/hal-00610714}

Preprint submitted on 23 Jul 2011

HAL is a multi-disciplinary open access archive for the deposit and dissemination of scientific research documents, whether they are published or not. The documents may come from teaching and research institutions in France or abroad, or from public or private research centers.
L'archive ouverte pluridisciplinaire HAL, est destinée au dépôt et à la diffusion de documents scientifiques de niveau recherche, publiés ou non, émanant des établissements d'enseignement et de recherche français ou étrangers, des laboratoires publics ou privés. 


\title{
THE QUADRATIC DYNATOMIC CURVES ARE SMOOTH AND IRREDUCIBLE
}

\author{
BUFF XAVIER AND TAN LEI \\ Dedicated to John Milnor's 80'th birthday
}

\begin{abstract}
We reprove here the smoothness and the irreducibility of the quadratic dynatomic curves $\left\{(c, z) \in \mathbb{C}^{2} \mid z\right.$ is $n$-periodic for $\left.z^{2}+c\right\}$.

The smoothness is due to Douady-Hubbard. Our proof here is based on elementary calculations on the pushforwards of specific quadratic differentials, following Thurston and Epstein. This approach is a computational illustration of the power of the far more general transversality theory of Epstein.

The irreducibility is due to Bousch, Morton and Lau-Schleicher with different approaches. Our proof is inspired by the proof of Lau-Schleicher. We use elementary combinatorial properties of the kneading sequences instead of internal addresses.
\end{abstract}

\section{INTRODUCTION}

For $c \in \mathbb{C}$, let $f_{c}: \mathbb{C} \rightarrow \mathbb{C}$ be the quadratic polynomial

$$
f_{c}(z):=z^{2}+c
$$

A point $z \in \mathbb{C}$ is periodic for $f_{c}$ if $f_{c}^{\circ n}(z)=z$ for some integer $n \geq 1$; it is of period $n$ if $f_{c}^{\circ k}(z) \neq z$ for $0<k<n$. For $n \geq 1$, set

$$
X_{n}:=\left\{(c, z) \in \mathbb{C}^{2} \mid z \text { is periodic of period } n \text { for } f_{c}\right\} .
$$

The objective of this note is to give new proofs of the following known results.

Theorem 1.1 (Douady-Hubbard). For every $n \geq 1$, the closure of $X_{n}$ in $\mathbb{C}^{2}$ is a smooth affine curve.

Theorem 1.2 (Bousch, Morton and Lau-Schleicher). For every $n \geq 1$ the closure of $X_{n}$ in $\mathbb{C}^{2}$ is irreducible.

Milnor [Mi2 reformulated in the language of quadratic differentials a proof of Tsujii showing that the topological entropy of the real quadratic polynomial $x \mapsto x^{2}+c$ varies monotonically with respect to the parameter $c$. Our approach here to prove Theorem 1.1 is similar. We use elementary calculations on quadratic differentials and Thurston's contraction principle (instead of parabolic implosion techniques used in the original proof of Douady-Hubbard). Our calculation is a computational illustration of the far deeper and more conceptual transversality theory of Epstein [E].

Theorem 1.2 has been proved by Bousch [B] and by Morton [Mo] using a combination of algebraic and dynamical arguments, and by Lau and Schleicher [LS, Sc] using dynamical

Date: July 23, 2011.

The first author is partially supported by the Institut Universitaire de France. 
arguments only. Our approach here follows essentially [LS, Sc], except we replace their argument on internal addresses [Sc, Lemma 4.5] by a purely combinatorial argument on kneading sequences (Lemma 4.2 below). Also, we make use of a result of Petersen-Ryd $[\mathrm{PR}]$ instead of Douady-Hubbard's parabolic implosion theory.

The somewhat similar curve consisting of cubic polynomial maps with periodic critical orbit is smooth of known Euler characteristic, due to the works of Milnor and BonifantKiwi-Milnor ([Mi3, BKM] $)$. But the irreducibility question remains open.

In Section 2 we prove that $\bar{X}_{n}$ is an affine curve by introducing dynatomic polynomials defining the curve, in Section 3 we prove the smoothness while in Section 4 we prove the irreducibility. Sections 3 and 4 can be read independently.

Acknowlegements. We thank Adam Epstein and John Milnor for helpful discussions, and William Thurston for his encouragement.

\section{Dynatomic polynomials}

In this section, we define the dynatomic polynomials $Q_{n} \in \mathbb{Z}[c, z]$ (see [Mi1] and [Si]) and show that

$$
\bar{X}_{n}=\left\{(c, z) \in \mathbb{C}^{2} \mid Q_{n}(c, z)=0\right\} .
$$

For $n \geq 1$, let $P_{n} \in \mathbb{Z}[c, z]$ be the polynomial defined by

$$
P_{n}(c, z):=f_{c}^{\circ n}(z)-z .
$$

The dynatomic polynomials $Q_{n}$ will be defined so that

$$
P_{n}=\prod_{k \mid n} Q_{k}
$$

Example 1. For $n=1$ and $n=2$, we have

$$
\begin{array}{ll}
P_{1}(c, z)=z^{2}-z+c, & P_{2}(c, z)=z^{4}+2 c z^{2}-z+c^{2}+c, \\
Q_{1}(c, z)=z^{2}-z+c, & Q_{2}(c, z)=z^{2}+z+c+1 \\
P_{1}(c, z)=Q_{1}(c, z), & P_{2}(c, z)=Q_{1}(c, z) \cdot Q_{2}(c, z) .
\end{array}
$$

Further examples may be found in [Si, Table 4.1].

With an abuse of notation, we will identify polynomials in $\mathbb{Z}[c, z]$ and polynomials in $\mathbf{Z}[z]$ with $\mathbf{Z}=\mathbb{Z}[c]$. In particular, we shall write $R(c, z)$ when $R \in \mathbf{Z}[z]$. Note that $P_{n} \in \mathbf{Z}[z]$ is a monic polynomial (its leading coefficient is 1 ) of degree $2^{n}$.

Proposition 2.1. There exists a unique sequence of monic polynomials $\left(Q_{n} \in \mathbf{Z}[z]\right)_{n \geq 1}$, such that for all $n \geq 1$, we have $P_{n}=\prod_{k \mid n} Q_{k}$.

Proof. The proof goes by induction on $n$. For $n=1$, it is necessary and sufficient to define

$$
Q_{1}(c, z):=P_{1}(c, z)=z^{2}-z+c \text {. }
$$


Note that $Q_{1} \in \mathbf{Z}[z]$ is indeed monic. Assume now that $n>1$ and that the polynomials $Q_{k}$ are defined for $1 \leq k<n$. Set

$$
A:=\prod_{k \mid n, k<n} Q_{k}
$$

Since the polynomials $Q_{k} \in \mathbf{Z}[z]$ are monic, the polynomial $A \in \mathbf{Z}[z]$ is also monic. So, we may perform a Euclidean division to find a monic quotient $Q \in \mathbf{Z}[z]$ and a remainder $R \in \mathbf{Z}[z]$ with degree $(R)<\operatorname{degree}(A)$, such that $P_{n}=Q A+R$. We need to show that $R=0$, which enables us to set $Q_{n}:=Q$.

Let $\Delta \in \mathbb{Z}[c]$ be the discriminant of $A$. We claim that $\Delta(0) \neq 0$. Indeed, for each $k<n$, the polynomial $Q_{k}(0, z) \in \mathbb{Z}[z]$ divides $P_{k}(0, z)=z^{2^{k}}-z$ whose roots are simple. So, the roots of $Q_{k}(0, z)$ are simple. In addition, a root $z_{0}$ of $Q_{k}(0, z)$ is a periodic point of $f_{0}$ whose period $m$ divides $k$. We have $m=k$ since otherwise,

$$
Q_{k}(0, z) \cdot P_{m}(0, z)=Q_{k}(0, z) \cdot \prod_{j \mid m} Q_{j}(0, z)
$$

would have a double root at $z_{0}$ and would at the same time divide

$$
P_{k}(0, z)=\prod_{j \mid k} Q_{j}(0, z)
$$

whose roots are simple. So, if $1 \leq k_{1}<k_{2}<n$, then $Q_{k_{1}}(0, z)$ and $Q_{k_{2}}(0, z)$ do not have common roots. This shows that the roots of $A(0, z)$ are simple, whence $\Delta(0) \neq 0$.

Fix $c_{0} \in \mathbb{C}$ such that $\Delta\left(c_{0}\right) \neq 0$ ( since $\Delta$ does not identically vanish, this holds for every $c_{0}$ outside a finite set). Then, the roots of $A\left(c_{0}, z\right) \in \mathbb{Z}[z]$ are simple. Such a root $z_{0}$ is a periodic point of $f_{c_{0}}$, with period dividing $n$, whence a root of $P_{n}\left(c_{0}, z\right)$. As a consequence, $A\left(c_{0}, z\right)$ divides $P_{n}\left(c_{0}, z\right)$ in $\mathbb{C}[z]$. It follows that $R\left(c_{0}, z\right)=0$ for all $z \in \mathbb{C}$. Since this is true for every $c_{0}$ outside a finite set, we have that $R=0$ as required.

Remark 1. The proof we gave shows that the dynatomic polynomials $Q_{n}$ have no repeated factors (otherwise $Q_{n}(0, z) \in \mathbb{Z}[z]$ would have a double root) and moreover, if $k_{1} \neq k_{2}$, then $Q_{k_{1}}$ and $Q_{k_{2}}$ do not have common factors (otherwise $Q_{k_{1}}(0, z) \in \mathbb{Z}[z]$ and $Q_{k_{2}}(0, z) \in \mathbb{Z}[z]$ would have a common root). Those facts will be used later.

Remark 2. The degree of $Q_{k}$ is at most that of $P_{k}$, that is $2^{k}$. It follows that the degree of $A:=\prod_{k \mid n, k<n} Q_{k}$ is at most $2^{n}-2$, and so, the degree of $Q_{n}=P_{n} / A$ is at least 2. In particular, for $n \geq 1$, the set $X_{n}$ is non-empty.

We extensively used the properties of roots of $Q_{n}(0, z) \in \mathbb{Z}[z]$. We will now study the properties of the roots of $Q_{n}\left(c_{0}, z\right) \in \mathbb{C}[z]$ for an arbitrary parameter $c_{0} \in \mathbb{C}$.

Proposition 2.2. Let $n \geq 1$ be a positive integer and $c_{0} \in \mathbb{C}$ be an arbitrary parameter. Then, $z_{0} \in \mathbb{C}$ is a root of $Q_{n}\left(c_{0}, z\right) \in \mathbb{C}[z]$ if and only if one of the following three exclusive conditions is satisfied:

(1) $z_{0}$ is periodic for $f_{c_{0}}$, the period is $n$ and the multiplier is not 1 ; in that case $Q_{n}\left(c_{0}, z\right)$ has a simple root at $z_{0}$, or 
(2) $z_{0}$ is periodic for $f_{c_{0}}$, the period is $n$ and the multiplier is equal to 1 ; in that case $Q_{n}\left(c_{0}, z\right)$ has a double root at $z_{0}$, or

(3) $z_{0}$ is periodic for $f_{c_{0}}$, the period $m<n$ is a proper divisor of $n$ and the multiplier of $z_{0}$ as a fixed point of $f_{c_{0}}^{\circ m}$ is a primitive $\frac{n}{m}$-th root of unity; in that case $Q_{n}\left(c_{0}, z\right)$ has a root of order $\frac{n}{m}$ at $z_{0}$.

Proof. If $Q_{n}\left(c_{0}, z_{0}\right)=0$, then $P_{n}\left(c_{0}, z_{0}\right)=0$ and so, $z_{0}$ is periodic for $f_{c_{0}}$ and the period $m$ divides $n$. Conversely, if $z_{0}$ is periodic of period $m$ for $f_{c_{0}}$, then $P_{k}\left(c_{0}, z_{0}\right)=0$ if and only if $k$ is a multiple of $m$. In particular, if $k$ is not a multiple of $m$, then $Q_{k}\left(c_{0}, z_{0}\right) \neq 0$. Since

$$
0=P_{m}\left(c_{0}, z_{0}\right)=\prod_{k \mid m} Q_{k}\left(c_{0}, z_{0}\right)
$$

we deduce that $Q_{m}\left(c_{0}, z_{0}\right)=0$.

Case 1. If the multiplier $\rho$ of $z_{0}$ as a fixed point of $f_{c_{0}}^{\circ m}$ is not a root of unity, then $P_{n}\left(c_{0}, z\right)$ has a simple root at $z_{0}$ whenever $n$ is a multiple of $m$. In that case, $Q_{m}\left(c_{0}, z\right)$ is a factor of $P_{n}\left(c_{0}, z\right)$ and so, no other factor of $P_{n}$ can vanish at $z_{0}$. As a consequence, $Q_{n}\left(c_{0}, z_{0}\right)$ vanishes if and only if $n=m$. In addition, $Q_{m}\left(c_{0}, z\right) \in \mathbb{C}[z]$ has a simple root at $z_{0}$.

Next, if the multiplier $\rho$ of $z_{0}$ as a fixed point of $f_{c_{0}}^{\circ m}$ is a primitive $s$-th root of unity, then the multiplier of $z_{0}$ as a fixed point of $f_{c_{0}}^{\circ m k}$ is $\rho^{k}$. It is equal to 1 if and only if $k$ is a multiple of $s$. In that case, $z_{0}$ is a multiple root of $P_{m k}\left(c_{0}, z\right)$ of order $s+1$. Indeed, $f_{c_{0}}$ has only one cycle of attracting petals since this cycle must attract the unique critical point of $f_{c_{0}}$.

Case 2. If $s=1$, then $P_{n}\left(c_{0}, z\right)$ has a double root at $z_{0}$ whenever $n$ is a multiple of $m$. As above, $Q_{n}\left(c_{0}, z_{0}\right)$ vanishes if and only if $n=m$, but this time, $Q_{m}\left(c_{0}, z\right) \in \mathbb{C}[z]$ has a double root at $z_{0}$.

Case 3. If $s \geq 2$, then $P_{n}\left(c_{0}, z\right)$ has a simple root at $z_{0}$ whenever $n$ is a multiple of $m$ but not a multiple of $m s$, and a multiple root at $z_{0}$ of order $s+1$ whenever $n$ is a multiple of $m s$. So, $Q_{n}\left(c_{0}, z_{0}\right)$ vanishes if and only if $n=m$ or $n=m s$; the polynomial $Q_{m}\left(c_{0}, z\right) \in \mathbb{C}[z]$ has a simple root at $z_{0}$ and the polynomial $Q_{m s}\left(c_{0}, z\right) \in \mathbb{C}[z]$ has a root of order $s$ at $z_{0}$.

Proposition 2.3. For all $n \geq 1$, we have

$$
\bar{X}_{n}=\left\{(c, z) \in \mathbb{C}^{2} \mid Q_{n}(c, z)=0\right\} .
$$

If $(c, z) \in \bar{X}_{n}-X_{n}$, then $z$ is periodic for $f_{c}$, its period $m$ is a proper divisor of $n$, and the multiplier of $z$ as a fixed point of $f_{c}^{\circ m}$ is a primitive $\frac{n}{m}$-th root of unity.

Proof. Let $Y_{n} \in \mathbb{C}^{2}$ be the affine curve defined by $Q_{n}$ :

$$
Y_{n}:=\left\{(c, z) \in \mathbb{C}^{2} \mid Q_{n}(c, z)=0\right\} .
$$

According to the previous Proposition,

- if $(c, z)$ belongs to $X_{n}$, then $Q_{n}(c, z)=0$ and so, $\bar{X}_{n} \subseteq Y_{n}$. 
THE QUADRATIC DYNATOMIC CURVES ARE SMOOTH AND IRREDUCIBLE

- if $(c, z) \in Y_{n}-X_{n}$, then $z$ is periodic for $f_{c}$, its period $m$ is a proper divisor of $n$, and the multiplier of $z$ as a fixed point of $f_{c}^{\circ m}$ is a primitive $\frac{n}{m}$-th root of unity. In particular, $Q_{m}(c, z)=0$. Since $Q_{n}$ and $Q_{m}$ do not have common factors, this only occurs for a finite set of points $(c, z) \in Y_{n}$. Thus, $Y_{n} \subseteq \bar{X}_{n}$.

Remark 3. We saw that $\bar{X}_{n}-X_{n}$ is finite. More generally, the set of points $\left(c_{0}, z_{0}\right) \in \bar{X}_{n}$ such that $f_{c_{0}}^{\circ n}\left(z_{0}\right)=z_{0}$ and $\left(f_{c_{0}}^{\circ n}\right)^{\prime}\left(z_{0}\right)=1$ is finite. Indeed, for such a point, $c_{0}$ is a root of the discriminant of $P_{n} \in \mathbf{Z}[z]$. Since the roots of $P_{n}(0, z)$ are simple, this discriminant does not vanish at $c=0$, and so, its roots form a finite set.

\section{Smoothness of The Dynatomic CURVES}

Our objective is now to give a proof of Theorem 1.1. We will prove the following more precise version. We shall denote by $\pi_{c}: \mathbb{C}^{2} \rightarrow \mathbb{C}$ the projection $(c, z) \mapsto c$ and by $\pi_{z}: \mathbb{C}^{2} \rightarrow \mathbb{C}$ the projection $(c, z) \mapsto z$.

Theorem 3.1. For every $n \geq 1$, the affine curve $\bar{X}_{n}$ is smooth. More precisely, for $\left(c_{0}, z_{0}\right) \in \bar{X}_{n}$, we have:

(1) if $z_{0} \in X_{n}$ has multiplier different from 1 , then $\pi_{c}: X_{n} \rightarrow \mathbb{C}$ is a local isomorphism;

(2) if $z_{0} \in X_{n}$ has multiplier 1 , then $\pi_{z}: X_{n} \rightarrow \mathbb{C}$ is a local isomorphism; in addition, $\pi_{c}: X_{n} \rightarrow \mathbb{C}$ has local degree 2 .

(3) if $z_{0} \in \bar{X}_{n}-X_{n}$ has multiplier a primitive $s$-th root of unity, then $\pi_{z}: \bar{X}_{n} \rightarrow \mathbb{C}$ is a local isomorphism; in addition, $\pi_{c}: \bar{X}_{n} \rightarrow \mathbb{C}$ has local degree $s$.

The idea is to apply the Implicit Function Theorem. In particular, we will prove that $\frac{\partial Q_{n}}{\partial z}\left(c_{0}, z_{0}\right) \neq 0$ in Case 1 (this is almost immediate), and that $\frac{\partial Q_{n}}{\partial c}\left(c_{0}, z_{0}\right) \neq 0$ in Cases 2 and 3 . This is where we have to work: following Epstein, we will first relate this partial derivative to the coefficient of a quadratic differential of the form $\left(f_{c_{0}}\right)_{*} \mathbf{q}-\mathbf{q}$; we will then show that $\left(f_{c_{0}}\right)_{*} \mathbf{q} \neq \mathbf{q}$ by using (a generalization of) Thurston's Contraction Principle. This approach is fundamentally different from Douady-Hubbard's original proof, where Fatou-Leau's flower theorem on parabolic periodic points as well as Douady-Hubbard's parabolic implosion theory play an essential role.

Once we know that in Cases 2 and 3 , the projection $\pi_{z}: X_{n} \rightarrow \mathbb{C}$ is a local isomorphism, the local degree of the projection $\pi_{c}: X_{n} \rightarrow \mathbb{C}$ follows from Proposition 2.2, Indeed, if $Q_{n}\left(c_{0}, z\right) \in \mathbb{C}[z]$ has a root of order $\nu$ at $z_{0}$ and if $\frac{\partial Q_{n}}{\partial c}\left(c_{0}, z_{0}\right) \neq 0$, then

$$
Q_{n}\left(c_{0}+\eta, z_{0}+\varepsilon\right)=a \eta+b \varepsilon^{\nu}+o(\eta)+o\left(\varepsilon^{\nu}\right) \quad \text { with } \quad a \neq 0 \text { and } b \neq 0
$$

and the solutions of $Q_{n}(c, z)=0$ are locally of the form $(c(z), z)$ with

$$
c\left(z_{0}+\varepsilon\right)=c_{0}-\frac{b}{a} \varepsilon^{\nu}+o\left(\varepsilon^{\nu}\right) .
$$

3.1. Case 1 of Theorem 3.1. Let $\left(c_{0}, z_{0}\right) \in X_{n}$ be such that the multiplier of $z_{0}$ as a fixed point of $f_{c_{0}}^{\text {on }}$ is not 1 . Then, $Q_{n}\left(c_{0}, z_{0}\right)=0$, for all $k<n, Q_{k}\left(c_{0}, z_{0}\right) \neq 0$ and 
$\left(f_{c_{0}}^{\circ n}\right)^{\prime}\left(z_{0}\right) \neq 1$. Since

$$
\prod_{k \mid n} Q_{k}(c, z)=P_{n}(c, z)
$$

we have

$$
\frac{\partial Q_{n}}{\partial z}\left(c_{0}, z_{0}\right) \cdot \prod_{k \mid n, k<n} Q_{k}\left(c_{0}, z_{0}\right)=\frac{\partial P_{n}}{\partial z}\left(c_{0}, z_{0}\right)=\left(f_{c_{0}}^{\circ n}\right)^{\prime}\left(z_{0}\right)-1 \neq 1
$$

As a consequence, $\frac{\partial Q_{k}}{\partial z}\left(c_{0}, z_{0}\right) \neq 0$. By the Implicit Function Theorem, $X_{n}$ is smooth near $\left(c_{0}, z_{0}\right)$ and the projection $\pi_{c}: X_{n} \rightarrow \mathbb{C}$ is a local isomorphism.

3.2. Case 2 of Theorem 3.1. Let $\left(c_{0}, z_{0}\right) \in X_{n}$ be such that the multiplier of $z_{0}$ as a fixed point of $f_{c_{0}}^{\text {on }}$ is 1 . As previously, since $Q_{n}\left(c_{0}, z_{0}\right)=0$ and

$$
\prod_{k \mid n} Q_{k}(c, z)=P_{n}(c, z)
$$

we have

$$
\frac{\partial Q_{n}}{\partial c}\left(c_{0}, z_{0}\right) \cdot \prod_{k \mid n, k<n} Q_{k}\left(c_{0}, z_{0}\right)=\frac{\partial P_{n}}{\partial c}\left(c_{0}, z_{0}\right) .
$$

Since for all $k<n, Q_{k}\left(c_{0}, z_{0}\right) \neq 0$ it is enough to prove that

$$
\frac{\partial P_{n}}{\partial c}\left(c_{0}, z_{0}\right) \neq 0
$$

We shall use the following notations: for $n \geq 0$, we let $\zeta_{n}: \mathbb{C} \rightarrow \mathbb{C}$ be defined by

$$
\zeta_{n}(c):=f_{c}^{\circ n}\left(z_{0}\right)
$$

and we set

$$
z_{n}:=\zeta_{n}\left(c_{0}\right)=f_{c_{0}}^{\circ n}\left(z_{0}\right) \quad \text { and } \quad \delta_{n}:=f_{c_{0}}^{\prime}\left(z_{n}\right)=2 z_{n}
$$

Since $P_{n}\left(c, z_{0}\right)=f_{c}^{\circ n}\left(z_{0}\right)-z_{0}=\zeta_{n}(c)-z_{0}$, we have

$$
\frac{\partial P_{n}}{\partial c}\left(c_{0}, z_{0}\right)=\zeta_{n}^{\prime}\left(c_{0}\right)
$$

Lemma 3.2 (Compare with [Mi2]). We have

$$
\zeta_{n}^{\prime}\left(c_{0}\right)=1+\delta_{n-1}+\delta_{n-1} \delta_{n-2}+\ldots+\delta_{n-1} \delta_{n-2} \cdots \delta_{1} .
$$

Proof. The function $\zeta_{0}$ is constant (equal to $z_{0}$ ). From $\zeta_{n}(c)=\left(\zeta_{n-1}(c)\right)^{2}+c$, we obtain

$$
\zeta_{n}^{\prime}\left(c_{0}\right)=1+\delta_{n-1} \zeta_{n-1}^{\prime}\left(c_{0}\right) \quad \text { with } \quad \zeta_{0}^{\prime}\left(c_{0}\right)=0
$$

The result follows by induction.

In order to prove that

$$
1+\delta_{n-1}+\delta_{n-1} \delta_{n-2}+\ldots+\delta_{n-1} \delta_{n-2} \cdots \delta_{1} \neq 0
$$

we shall now work with meromorphic quadratic differentials. 
3.2.1. Quadratic differentials. A meromorphic quadratic differential $\mathbf{q}$ on $\mathbb{C}$ takes the form $\mathbf{q}=q \mathrm{dz}^{2}$ with $q$ a meromorphic function on $\mathbb{C}$. We use $\mathcal{Q}(\mathbb{C})$ to denote the set of meromorphic quadratic differentials on $\mathbb{C}$ whose poles (if any) are all simple. If $\mathbf{q}=q \mathrm{dz}^{2} \in \mathcal{Q}(\mathbb{C})$ and $U$ is a bounded open subset of $\mathbb{C}$, the norm

$$
\|\mathbf{q}\|_{U}:=\iint_{U}|q(x+\mathrm{i} y)| \mathrm{d} x \mathrm{~d} y
$$

is well defined and finite.

\section{Example 2.}

$$
\left\|\frac{\mathrm{dz}^{2}}{z}\right\|_{D(0, R)}=\int_{0}^{2 \pi} \int_{0}^{R} \frac{1}{r} r \mathrm{~d} r \mathrm{~d} \theta=2 \pi R
$$

3.2.2. Pushforward. For $f: \mathbb{C} \rightarrow \mathbb{C}$ a non-constant polynomial and $\mathbf{q}=q \mathrm{dz}^{2}$ a meromorphic quadratic differential on $\mathbb{C}$, the pushforward $f_{*} \mathbf{q}$ is defined by

$$
f_{*} \mathbf{q}:=T q \mathrm{dz}^{2} \quad \text { with } \quad T q(z):=\sum_{f(w)=z} \frac{q(w)}{f^{\prime}(w)^{2}} .
$$

If $\mathbf{q} \in \mathcal{Q}(\mathbb{C})$, then $f_{*} \mathbf{q} \in \mathcal{Q}(\mathbb{C})$ also.

Lemma 3.3 (Compare with [Mi2] or [L]). For $f=f_{c}$, we have

$$
\left\{\begin{array}{l}
f_{*}\left(\frac{\mathrm{dz}^{2}}{z}\right)=0 \\
f_{*}\left(\frac{\mathrm{dz}^{2}}{z-a}\right)=\frac{1}{f^{\prime}(a)}\left(\frac{\mathrm{dz}^{2}}{z-f(a)}-\frac{\mathrm{dz}^{2}}{z-c}\right) \text { if } a \neq 0 .
\end{array}\right.
$$

Proof. If $f(w)=z$, then $w= \pm \sqrt{z-c}$ and

$$
\mathrm{dw}^{2}=\frac{\mathrm{dz}^{2}}{4(z-c)}
$$

We then have

$$
\begin{aligned}
f_{*}\left(\frac{\mathrm{dz}^{2}}{z-a}\right) & =\frac{\mathrm{dz}^{2}}{4(z-c)}\left(\frac{1}{\sqrt{z-c}-a}+\frac{1}{-\sqrt{z-c}-a}\right) \\
& =\frac{a \mathrm{dz}^{2}}{2(z-c)(z-f(a))} .
\end{aligned}
$$

If $a=0$, we get the first equality in (3.1). Otherwise, we get the second equality using

$$
\frac{1}{f^{\prime}(a)}\left(\frac{1}{z-f(a)}-\frac{1}{z-c}\right)=\frac{f(a)-c}{2 a(z-c)(z-f(a))}=\frac{a}{2(z-c)(z-f(a))} .
$$

3.2.3. A particular quadratic differential. Consider the quadratic differential $\mathbf{q} \in \mathcal{Q}(\mathbb{C})$ defined by

$$
\mathbf{q}:=\sum_{k=0}^{n-1} \frac{\rho_{k}}{z-z_{k}} \mathrm{dz}^{2} \quad \text { with } \quad \rho_{k}=\delta_{n-1} \delta_{n-2} \cdots \delta_{k}
$$


The multiplier of $z_{0}$ as a periodic point of $f_{c_{0}}$ is $\rho_{0}=1$. So,

$$
\frac{\rho_{n-1}}{\delta_{n-1}}=1=\rho_{0} \text {. }
$$

In addition, for $0 \leq k \leq n-2$, we have

$$
\frac{\rho_{k}}{\delta_{k}}=\rho_{k+1}
$$

Applying Lemma 3.3, and writing $f$ for $f_{c_{0}}$, we obtain

$$
f_{*} \mathbf{q}=\sum_{k=0}^{n-1} \frac{\rho_{k}}{\delta_{k}}\left(\frac{\mathrm{dz}^{2}}{z-z_{k+1}}-\frac{\mathrm{dz}^{2}}{z-c_{0}}\right)=\mathbf{q}-\left(\sum_{k=0}^{n-1} \rho_{k}\right) \cdot \frac{\mathrm{dz}^{2}}{z-c_{0}} .
$$

As mentioned earlier,

$$
\frac{\partial P_{n}}{\partial c}\left(c_{0}, z_{0}\right)=\zeta_{n}^{\prime}\left(c_{0}\right)=\sum_{k=0}^{n-1} \rho_{k}
$$

It is therefore enough to prove that $f_{*} \mathbf{q} \neq \mathbf{q}$. This is done in the next paragraph using a Contraction Principle.

3.2.4. Contraction Principle. The following lemma is a weak version of Thurston's contraction principle (which applies to the setting of rational maps on $\mathbb{P}^{1}$ ).

Lemma 3.4 (Contraction Principle). For a non-constant polynomial $f$ and a round disk $V$ of radius large enough so that $U:=f^{-1}(V)$ is relatively compact in $V$, we have

$$
\left\|f_{*} \mathbf{q}\right\|_{V} \leq\|\mathbf{q}\|_{U}<\|\mathbf{q}\|_{V}, \quad \forall \mathbf{q} \in \mathcal{Q}(\mathbb{C}) .
$$

Proof. The strict inequality on the right is a consequence of the fact that $U$ is relatively compact in $V$. The inequality on the left comes from

$$
\begin{aligned}
\left\|f_{*} \mathbf{q}\right\|_{V} & =\iint_{x+i y \in V}\left|\sum_{f(w)=x+i y} \frac{q(w)}{f^{\prime}(w)^{2}}\right| \mathrm{d} x \mathrm{~d} y \\
& \leq \iint_{x+i y \in V} \sum_{f(w)=x+i y}\left|\frac{q(w)}{f^{\prime}(w)^{2}}\right| \mathrm{d} x \mathrm{~d} y=\iint_{u+i v \in U}|q(u+i v)| \mathrm{d} u \mathrm{~d} v=\|\mathbf{q}\|_{U} .
\end{aligned}
$$

Corollary 3.5. If $f: \mathbb{C} \rightarrow \mathbb{C}$ is a polynomial and if $\mathbf{q} \in \mathcal{Q}(\mathbb{C})$, then $f_{*} \mathbf{q} \neq \mathbf{q}$.

3.3. Case 3 of Theorem 3.1. Let $\left(c_{0}, z_{0}\right) \in \bar{X}_{n}-X_{n}$ be such that $z_{0}$ is periodic for $f_{c_{0}}$ with period $m<n$ dividing $n$ and multiplier $\rho$, a primitive $s$-th root of unity with $s:=\frac{n}{m}$.

According to Proposition 2.2 point 3 , the polynomial $Q_{n}\left(c_{0}, z\right) \in \mathbb{C}[z]$ has a root of order $s \geq 2$ at $z_{0}$, so that

$$
\frac{\partial Q_{n}}{\partial z}\left(c_{0}, z_{0}\right)=0
$$

We want to show that

$$
\frac{\partial Q_{n}}{\partial c}\left(c_{0}, z_{0}\right) \neq 0
$$


Let us write

$$
P_{n}(c, z)=P_{m}(c, z) \cdot R(c, z) \quad \text { with } \quad R(c, z)=\prod_{k \mid n, k \nmid m} Q_{k}(c, z) .
$$

On the first hand, since $Q_{n}\left(c_{0}, z_{0}\right)=0$, we have

$$
\frac{\partial R}{\partial c}\left(c_{0}, z_{0}\right)=\frac{\partial Q_{n}}{\partial c}\left(c_{0}, z_{0}\right) \cdot \prod_{k \mid n, k \nmid m, k<n} Q_{k}\left(c_{0}, z_{0}\right) .
$$

Since for all $k<n$ with $k \neq m, Q_{k}\left(c_{0}, z_{0}\right) \neq 0$, it is enough to prove that

$$
\frac{\partial R}{\partial c}\left(c_{0}, z_{0}\right) \neq 0 \text {. }
$$

3.3.1. Variation along $X_{m}$. Note that $\left(c_{0}, z_{0}\right) \in X_{m}$ and the multiplier $\rho$ of $z_{0}$ as a fixed point of $f_{c_{0}}^{\circ m}$ is $\rho \neq 1$. Thus, according to Case $1, X_{m}$ is locally the graph of a function $\zeta(c)$ defined and holomorphic near $c_{0}$ with $\zeta\left(c_{0}\right)=z_{0}$. The point $\zeta(c)$ is periodic of period $m$ for $f_{c}$. We denote by $\rho_{c}$ its multiplier and set

$$
\dot{\rho}:=\left.\frac{d \rho_{c}}{d c}\right|_{c_{0}} .
$$

Lemma 3.6. We have

$$
\frac{\partial R}{\partial c}\left(c_{0}, z_{0}\right)=\frac{s \dot{\rho}}{\rho(\rho-1)}
$$

Proof. Differentiating Equation $\sqrt{3.2}$ with respect to $z$, and evaluating at $(c, \zeta(c))$, we get:

$$
\rho_{c}^{s}-1=\left(\rho_{c}-1\right) \cdot R(c, \zeta(c))+\underbrace{P_{m}(c, \zeta(c))}_{=0} \cdot \frac{\partial R}{\partial z}(c, \zeta(c))=\left(\rho_{c}-1\right) \cdot R(c, \zeta(c)) .
$$

Differentiating with respect to $c$ and evaluating at $c_{0}$, we get:

$$
s \rho^{s-1} \dot{\rho}=\dot{\rho} \underbrace{R\left(c_{0}, z_{0}\right)}_{=0}+(\rho-1) \frac{\partial R}{\partial c}\left(c_{0}, z_{0}\right)+(\rho-1) \underbrace{\frac{\partial R}{\partial z}\left(c_{0}, z_{0}\right)}_{=0} \zeta^{\prime}\left(c_{0}\right)=(\rho-1) \frac{\partial R}{\partial c}\left(c_{0}, z_{0}\right) .
$$

The result follows since $\rho^{s}=1$ and so, $\rho^{s-1}=1 / \rho$.

Thus, we are left with proving that $\dot{\rho} \neq 0$. This will be done by using a particular meromorphic quadratic differential having double poles along the cycle of $z_{0}$.

\subsubsection{Quadratic differentials with double poles.}

Lemma 3.7 (Compare with $[\mathrm{L}]$ ). For $f=f_{c}$, we have

$$
f_{*}\left(\frac{\mathrm{dz}^{2}}{(z-a)^{2}}\right)=\frac{\mathrm{dz}^{2}}{(z-f(a))^{2}}-\frac{1}{2 a^{2}}\left(\frac{\mathrm{dz}^{2}}{z-f(a)}-\frac{\mathrm{dz}^{2}}{z-c}\right) \text { if } a \neq 0 .
$$


Proof. If $f(w)=z$, then $w= \pm \sqrt{z-c}$ and

$$
\mathrm{dw}^{2}=\frac{\mathrm{dz}^{2}}{4(z-c)} .
$$

Then

$$
\begin{aligned}
f_{*}\left(\frac{\mathrm{dz}^{2}}{(z-a)^{2}}\right) & =\frac{\mathrm{dz}^{2}}{4(z-c)}\left(\frac{1}{(\sqrt{z-c}-a)^{2}}+\frac{1}{(-\sqrt{z-c}-a)^{2}}\right) \\
& =\frac{\left(z-c+a^{2}\right) \mathrm{dz}^{2}}{2(z-c)\left(z-c-a^{2}\right)^{2}}=\frac{\left(z-c+a^{2}\right) \mathrm{dz}^{2}}{2(z-c)(z-f(a))^{2}} .
\end{aligned}
$$

Decomposing the last expression into partial fractions gives

$$
\frac{z-c+a^{2}}{2(z-c)(z-f(a))^{2}}=\frac{A}{(z-f(a))^{2}}+\frac{B}{z-f(a)}+\frac{C}{z-c}
$$

with

and

$$
A=\frac{f(a)-c+a^{2}}{2(f(a)-c)}=\frac{2 a^{2}}{2 a^{2}}=1, \quad C=\frac{c-c+a^{2}}{2(c-f(a))^{2}}=\frac{a^{2}}{2 a^{4}}=\frac{1}{2 a^{2}}
$$

$$
B=-C=-\frac{1}{2 a^{2}}
$$

Set $f:=f_{c_{0}}$,

$$
z_{k}:=f^{\circ k}\left(z_{0}\right), \quad \delta_{k}:=f^{\prime}\left(z_{k}\right)=2 z_{k}, \quad \zeta_{k}(c):=f_{c}^{\circ k}(\zeta(c)) \quad \text { and } \quad \dot{\zeta}_{k}:=\zeta_{k}^{\prime}\left(c_{0}\right) .
$$

Then

Since

$$
\zeta_{k+1}(c)=f_{c}\left(\zeta_{k}(c)\right) \quad \text { and } \quad \zeta_{n}=\zeta_{0} .
$$

$$
\delta_{0} \delta_{1} \cdots \delta_{m-1}=\rho \neq 1,
$$

there is a unique $m$-tuple $\left(\mu_{0}, \ldots, \mu_{m-1}\right)$ such that

$$
\mu_{k+1}=\frac{\mu_{k}}{2 z_{k}}-\frac{1}{2 z_{k}^{2}}
$$

where the indices are considered to be modulo $m$.

Now consider the quadratic differential $\mathbf{q}$ (with double poles) defined by

$$
\mathbf{q}:=\sum_{k=0}^{m-1}\left(\frac{1}{\left(z-z_{k}\right)^{2}}+\frac{\mu_{k}}{z-z_{k}}\right) \mathrm{dz}^{2} .
$$

Lemma 3.8 (Levin). We have

$$
f_{*} \mathbf{q}=\mathbf{q}-\frac{\dot{\rho}}{\rho} \cdot \frac{\mathrm{dz}^{2}}{z-c_{0}} .
$$

Proof. By construction of $\mathbf{q}$ and the calculation of $f_{*} \mathbf{q}$ in Lemma 3.3, the polar parts of $\mathbf{q}$ and $f_{*} \mathbf{q}$ along the cycle of $z_{0}$ are identical. But $f_{*} \mathbf{q}$ has an extra simple pole at the critical value $c_{0}$ with coefficient

$$
\sum_{k=0}^{m-1}\left(-\frac{\mu_{k}}{2 z_{k}}+\frac{1}{2 z_{k}^{2}}\right)=-\sum_{k=0}^{m-1} \mu_{k+1} .
$$


We need to show that this coefficient is equal to $-\frac{\dot{\rho}}{\rho}$.

Using $\zeta_{k+1}(c)=\left(\zeta_{k}(c)\right)^{2}+c$, we get

$$
\dot{\zeta}_{k+1}=2 z_{k} \dot{\zeta}_{k}+1
$$

It follows that

$$
\dot{\zeta}_{k+1} \mu_{k+1}-\mu_{k+1}=2 z_{k} \dot{\zeta}_{k} \mu_{k+1}=\dot{\zeta}_{k} \mu_{k}-\frac{\dot{\zeta}_{k}}{z_{k}}
$$

Therefore

$$
\sum_{k=0}^{m-1} \mu_{k+1}=\sum_{k=0}^{m-1}\left(\dot{\zeta}_{k+1} \mu_{k+1}-\dot{\zeta}_{k} \mu_{k}+\frac{\dot{\zeta}_{k}}{z_{k}}\right)=\sum_{k=0}^{m-1} \frac{\dot{\zeta}_{k}}{z_{k}}=\frac{\dot{\rho}}{\rho},
$$

where last equality is obtained by evaluating at $c_{0}$ of the logarithmic derivative of

$$
\rho_{c}:=\prod_{k=0}^{m-1} 2 \zeta_{k}(c)
$$

To complete the proof that $\dot{\rho} \neq 0$, we will use a generalization of the Contraction Principle due to Epstein.

Lemma 3.9 (Epstein). We have $f_{*} \mathbf{q} \neq \mathbf{q}$.

Proof. The proof rests again on the contraction principle, but we can not apply directly Lemma 3.4 since $\mathbf{q}$ is not integrable near the cycle $\left\langle z_{0}, \ldots, z_{m-1}\right\rangle$. Consider a sufficiently large round disk $V$ so that $U:=f^{-1}(V)$ is relatively compact in $V$. Given $\varepsilon>0$, we set

$$
V_{\varepsilon}:=\bigcup_{k=1}^{m} f^{\circ k}\left(D\left(z_{0}, \varepsilon\right)\right) \text { and } U_{\varepsilon}:=f^{-1}\left(V_{\varepsilon}\right)
$$

When $\varepsilon$ tends to 0 , we have

$$
\left\|f_{*} \mathbf{q}\right\|_{V-V_{\varepsilon}} \leq\|\mathbf{q}\|_{U-U_{\varepsilon}}=\|\mathbf{q}\|_{V-V_{\varepsilon}}-\|\mathbf{q}\|_{V-U}+\|\mathbf{q}\|_{U_{\varepsilon}-V_{\varepsilon}}-\|\mathbf{q}\|_{V_{\varepsilon}-U_{\varepsilon}}
$$

If we had $f_{*} \mathbf{q}=\mathbf{q}$, we would have

$$
0<\|\mathbf{q}\|_{V-U} \leq\|\mathbf{q}\|_{U_{\varepsilon}-V_{\varepsilon}}
$$

However, $\|\mathbf{q}\|_{U_{\varepsilon}-V_{\varepsilon}}$ tends to 0 as $\varepsilon$ tends to 0 , which is a contradiction. Indeed, $\mathbf{q}=q \mathrm{dz}^{2}$, the meromorphic function $q$ being equivalent to $\frac{1}{\left(z-z_{0}\right)^{2}}$ as $z$ tends to $z_{0}$. In addition, since the multiplier of $z_{0}$ has modulus 1 ,

$$
D\left(z_{0}, \varepsilon\right) \subset U_{\varepsilon}-V_{\varepsilon} \subset D\left(z_{0}, \varepsilon^{\prime}\right) \quad \text { with } \quad \frac{\varepsilon^{\prime}}{\varepsilon} \underset{\varepsilon \rightarrow 0}{\longrightarrow} 1 .
$$

Therefore,

$$
\|\mathbf{q}\|_{U_{\varepsilon}-V_{\varepsilon}} \leq \int_{0}^{2 \pi} \int_{\varepsilon}^{\varepsilon^{\prime}} \frac{1+o(1)}{r^{2}} r \mathrm{~d} r \mathrm{~d} \theta=2 \pi(1+o(1)) \log \frac{\varepsilon^{\prime}}{\varepsilon} \underset{\varepsilon \rightarrow 0}{\longrightarrow} 0 .
$$




\section{IRREDUCIBILITy OF THE DYNATOMIC CURVES}

Our objective is now to give a proof of Theorem 1.2. Note that since the affine curve $\bar{X}_{n}$ is defined by a polynomial $Q_{n}$ which has no repeated factors, this proves that $Q_{n}$ is irreducible.

Since $\bar{X}_{n}$ is smooth, we may equivalently prove the following result.

Theorem 4.1. For every $n \geq 1$, the set $\bar{X}_{n}$ is connected.

4.1. Kneading sequences. Set $\mathbb{T}=\mathbb{R} / \mathbb{Z}$ and let $\tau: \mathbb{T} \rightarrow \mathbb{T}$ be the angle doubling map

$$
\tau: \mathbb{T} \ni \theta \mapsto 2 \theta \in \mathbb{T} \text {. }
$$

By abuse of notation we shall often identify an angle $\theta \in \mathbb{T}$ with its representative in [0,1[. In particular, the angle $\theta / 2 \in \mathbb{T}$ is the element of $\tau^{-1}(\theta)$ with representative in $\left[0,1 / 2\left[\right.\right.$ and the angle $(\theta+1) / 2$ is the element of $\tau^{-1}(\theta)$ with representative in $[1 / 2,1[$.

Every angle $\theta \in \mathbb{T}$ has an associated kneading sequence $\nu(\theta)=\nu_{1} \nu_{2} \nu_{3} \ldots$ defined by

$$
\nu_{k}=\left\{\begin{array}{l}
\left.1 \quad \text { if } \tau^{\circ(k-1)}(\theta) \in\right] \frac{\theta}{2}, \frac{\theta+1}{2}[, \\
0 \quad \text { if } \tau^{\circ(k-1)}(\theta) \in \mathbb{T}-\left[\frac{\theta}{2}, \frac{\theta+1}{2}\right], \\
\star \quad \text { if } \tau^{\circ(k-1)}(\theta) \in\left\{\frac{\theta}{2}, \frac{\theta+1}{2}\right\} .
\end{array}\right.
$$

For example, $\nu(1 / 7)=\overline{11 \star}$ and $\nu(7 / 31)=\overline{1100 \star}$.

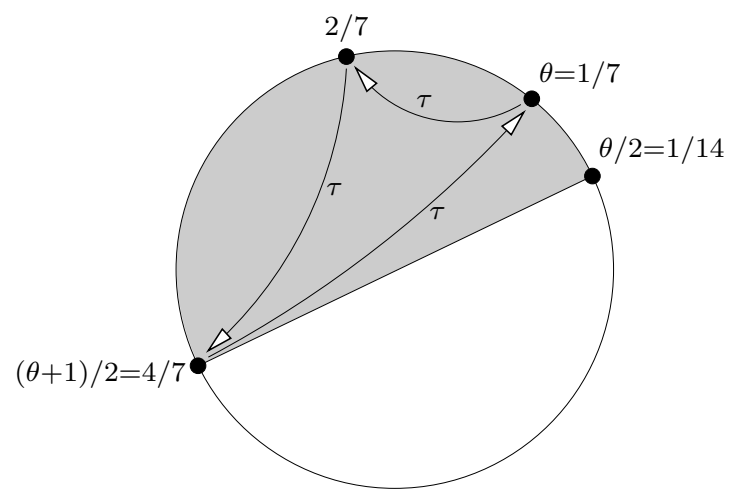

Figure 1 . The kneading sequence of $\theta=1 / 7$ is $\nu(1 / 7)=\overline{11 \star}$.

We shall say that an angle $\theta \in \mathbb{T}$, periodic under $\tau$, is maximal in its orbit if its representative in $[0,1)$ is maximal among the representatives of $\tau^{\circ j}(\theta)$ in $[0,1)$ for all $j \geq 1$. If the period is $n$ and the binary expansion of $\theta$ is.$\overline{\varepsilon_{1} \ldots \varepsilon_{n}}$, then $\theta$ is maximal in its orbit if and only if the periodic sequence $\overline{\varepsilon_{1} \ldots \varepsilon_{n}}$ is maximal (in the lexigographic order) among its iterated shifts, where the shift of a sequence $\varepsilon_{1} \varepsilon_{2} \varepsilon_{3}$ indexed by $\mathbb{N}$ is

$$
\sigma\left(\varepsilon_{1} \varepsilon_{2} \varepsilon_{3} \ldots\right)=\varepsilon_{2} \varepsilon_{3} \varepsilon_{4} \ldots
$$

Example 3. The angle $7 / 31=. \overline{00111}$ is not maximal in its orbit but $28 / 31=. \overline{11100}$ is maximal in the same orbit. 
The following lemma indicates cases where the binary expansion and the kneading sequence coincide.

Lemma 4.2. Let $\theta \in \mathbb{T}$ be a periodic angle which is maximal in its orbit and let $\overline{\varepsilon_{1} \ldots \varepsilon_{n}}$ be its binary expansion. Then, $\varepsilon_{n}=0$ and the kneading sequence $\nu(\theta)$ is equal to $\overline{\varepsilon_{1} \ldots \varepsilon_{n-1} \star}$.

For example,

$$
\frac{28}{31}=\overline{11100} \text { and } \quad \nu(\theta)=\overline{1110 \star} .
$$

Proof. Since $\theta$ is maximal in its orbit under $\tau$, the orbit of $\theta$ is disjoint from $] \theta / 2,1 / 2] \cup] \theta, 1]$. It follows that the orbit $\tau^{\circ j}(\theta), j=0,1, \ldots, n-2$ have the same itinerary relative to the two partitions $\mathbb{T}-\left\{0, \frac{1}{2}\right\}$ and $\mathbb{T}-\left\{\frac{\theta}{2}, \frac{\theta+1}{2}\right\}$. The first one gives the binary expansion whereas the second gives the kneading sequence. Therefore, the kneading sequence of $\theta$ is $\overline{\varepsilon_{1} \ldots \varepsilon_{n-1} \star}$. Since $\tau^{\circ(n-1)}(\theta) \in \tau^{-1}(\theta)=\left\{\frac{\theta}{2}, \frac{\theta+1}{2}\right\}$ and since $\left.\left.\frac{\theta+1}{2} \in\right] \theta, 1\right]$, we must have $\tau^{\circ(n-1)}(\theta)=\frac{\theta}{2}<\frac{1}{2}$. So $\varepsilon_{n}$, as the first digit of $\tau^{\circ(n-1)}(\theta)$, must be equal to 0 .

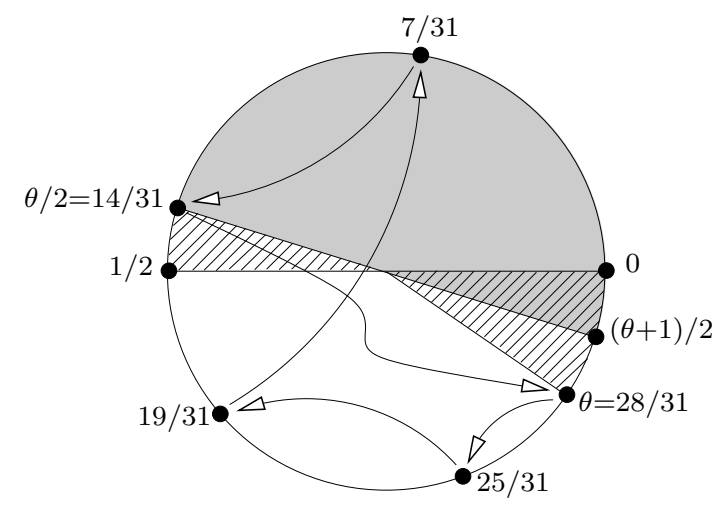

Figure 2. The kneading sequence of $\theta:=28 / 31=. \overline{11100}$ is $\nu(28 / 31)=\overline{1110 \star}$.

4.2. Filled-in Julia sets and the Mandelbrot set. We will use results proved by Douady and Hubbard in the Orsay Notes [DH] (see also [PR] for simpler proofs of some results) that we now recall.

For $c \in \mathbb{C}$, we denote by $K_{c}$ the filled-in Julia set of $f_{c}$, that is the set of points $z \in \mathbb{C}$ whose orbit under $f_{c}$ is bounded. We denote by $M$ the Mandelbrot set, that is the set of parameters $c \in \mathbb{C}$ for which the critical point 0 belongs to $K_{c}$.

If $c \in M$, then $K_{c}$ is connected. There is a conformal isomorphism $\phi_{c}: \mathbb{C}-K_{c} \rightarrow \mathbb{C}-\overline{\mathbb{D}}$ which satisfies $\phi_{c} \circ f_{c}=f_{0} \circ \phi_{c}$. The dynamical ray of angle $\theta \in \mathbb{T}$ is

$$
R_{c}(\theta):=\left\{z \in \mathbb{C}-K_{c} \mid \arg \left(\phi_{c}(z)\right)=2 \pi \theta\right\} .
$$

If $\theta$ is rational, then as $r$ tends to 1 from above, $\phi_{c}^{-1}\left(r \mathrm{e}^{2 \pi i \theta}\right)$ converges to a point $\gamma_{c}(\theta) \in K_{c}$. We say that $R_{c}(\theta)$ lands at $\gamma_{c}(\theta)$. We have $f_{c} \circ \gamma_{c}=\gamma_{c} \circ \tau$ on $\mathbb{Q} / \mathbb{Z}$. In particular, if $\theta$ is periodic under $\tau$, then $\gamma_{c}(\theta)$ is periodic under $f_{c}$. In addition, $\gamma_{c}(\theta)$ is either repelling (its multiplier has modulus $>1$ ) or parabolic (its multiplier is a root of unity). 
If $c \notin M$, then $K_{c}$ is a Cantor set. There is a conformal isomorphism $\phi_{c}: U_{c} \rightarrow V_{c}$ between neighborhoods of $\infty$ in $\mathbb{C}$, which satisfies $\phi_{c} \circ f_{c}=f_{0} \circ \phi_{c}$ on $U_{c}$. We may choose $U_{c}$ so that $U_{c}$ contains the critical value $c$ and $V_{c}$ is the complement of a closed disk. For each $\theta \in \mathbb{T}$, there is an infimum $r_{c}(\theta) \geq 1$ such that $\phi_{c}^{-1}$ extends analytically along $R_{0}(\theta) \cap\left\{z \in \mathbb{C}\left|r_{c}(\theta)<\right| z \mid\right\}$. We denote by $\psi_{c}$ this extension and by $R_{c}(\theta)$ the dynamical ray

$$
R_{c}(\theta):=\psi_{c}\left(R_{0}(\theta) \cap\left\{z \in \mathbb{C}\left|r_{c}(\theta)<\right| z \mid\right\}\right) .
$$

As $r$ tends to $r_{c}(\theta)$ from above, $\psi_{c}\left(r \mathrm{e}^{2 \pi i \theta}\right)$ converges to a point $x \in \mathbb{C}$. If $r_{c}(\theta)>1$, then $x \in \mathbb{C}-K_{c}$ is an iterated preimage of 0 and we say that $R_{c}(\theta)$ bifucates at $x$. If $r_{c}(\theta)=1$, then $\gamma_{c}(\theta):=x$ belongs to $K_{c}$ and we say that $R_{c}(\theta)$ lands at $\gamma_{c}(\theta)$. Again, $f_{c} \circ \gamma_{c}=\gamma_{c} \circ \tau$ on the set of $\theta$ such that $R_{c}(\theta)$ does not bifurcate. In particular, if $\theta$ is periodic under $\tau$ and $R_{c}(\theta)$ does not bifurcate, then $\gamma_{c}(\theta)$ is periodic under $f_{c}$.

The Mandelbrot set is connected. The map

$$
\phi_{M}: \mathbb{C}-M \ni c \mapsto \phi_{c}(c) \in \mathbb{C}-\overline{\mathbb{D}}
$$

is a conformal isomorphism. For $\theta \in \mathbb{T}$, the parameter ray $R_{M}(\theta)$ is

$$
R_{M}(\theta):=\left\{c \in \mathbb{C}-M \mid \arg \left(\phi_{M}(c)\right)=2 \pi \theta\right\} .
$$

It is known that if $\theta$ is rational, then as $r$ tends to 1 from above, $\phi_{M}^{-1}\left(r \mathrm{e}^{2 \pi i \theta}\right)$ converges to a point $\gamma_{M}(\theta) \in M$. We say that $R_{M}(\theta)$ lands at $\gamma_{M}(\theta)$.
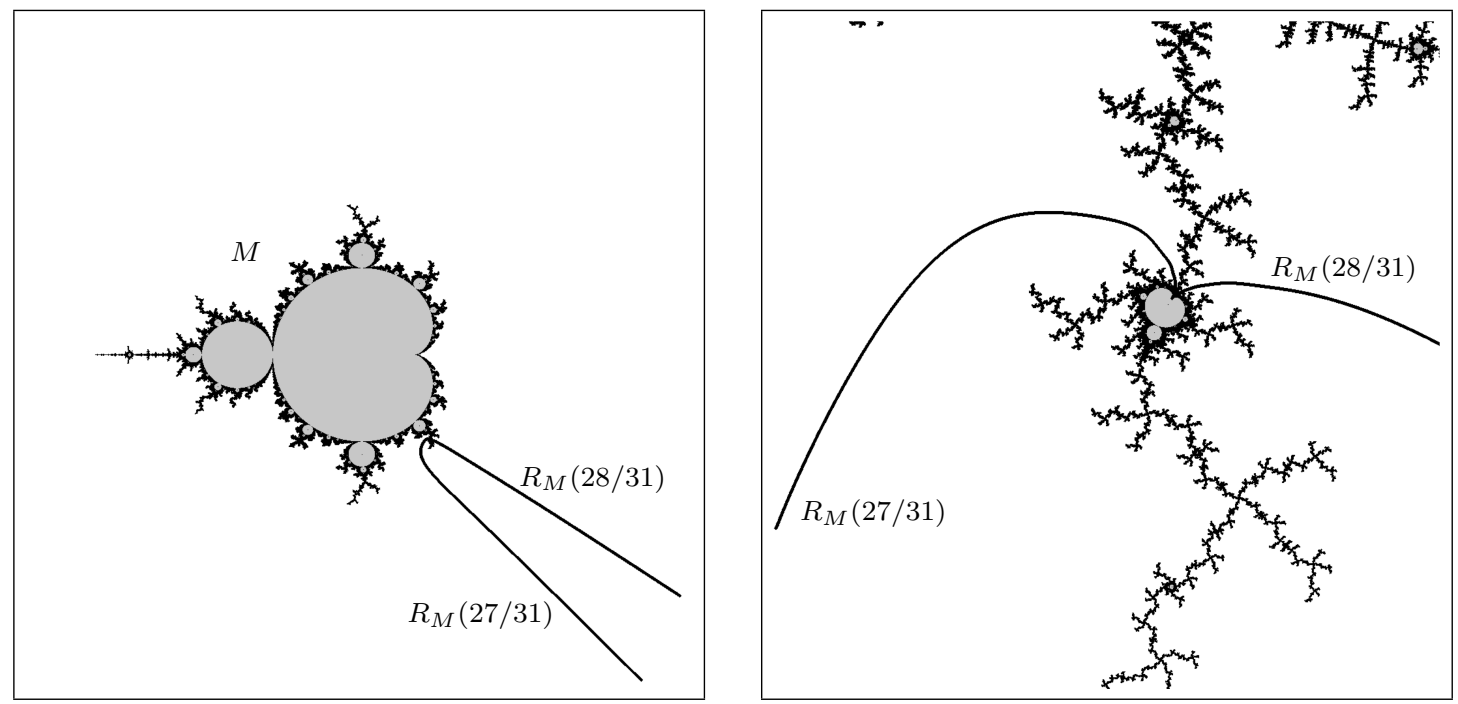

FiguRE 3 . The parameter rays $R_{M}(27 / 31)$ and $R_{M}(28 / 31)$ land at a common root of a primitive hyperbolic component.

If $\theta$ is periodic for $\tau$ of exact period $n$ and if $c=\gamma_{M}(\theta)$, then the point $\gamma_{c}(\theta)$ is periodic for $f_{c}$ with period dividing $n$ and multiplier a root of unity. If the period of $\gamma_{c}(\theta)$ for $f_{c}$ is exactly $n$ then the multiplier is $1, \gamma_{c}(\theta)$ disconnects $K_{c}$ in exactly two connected components and $c$ is the root of a primitive hyperbolic component of $M$.

The parameter ray $R_{M}(0)$ lands at $1 / 4$ and this is the only ray landing at $1 / 4$. Let us now assume that $c \in \mathbb{C}-\{1 / 4\}$ is the root of a hyperbolic component of $M$, that is $f_{c}$ has a parabolic cycle. Then there are exactly two parameter rays $R_{M}(\theta)$ and $R_{M}(\eta)$ landing 

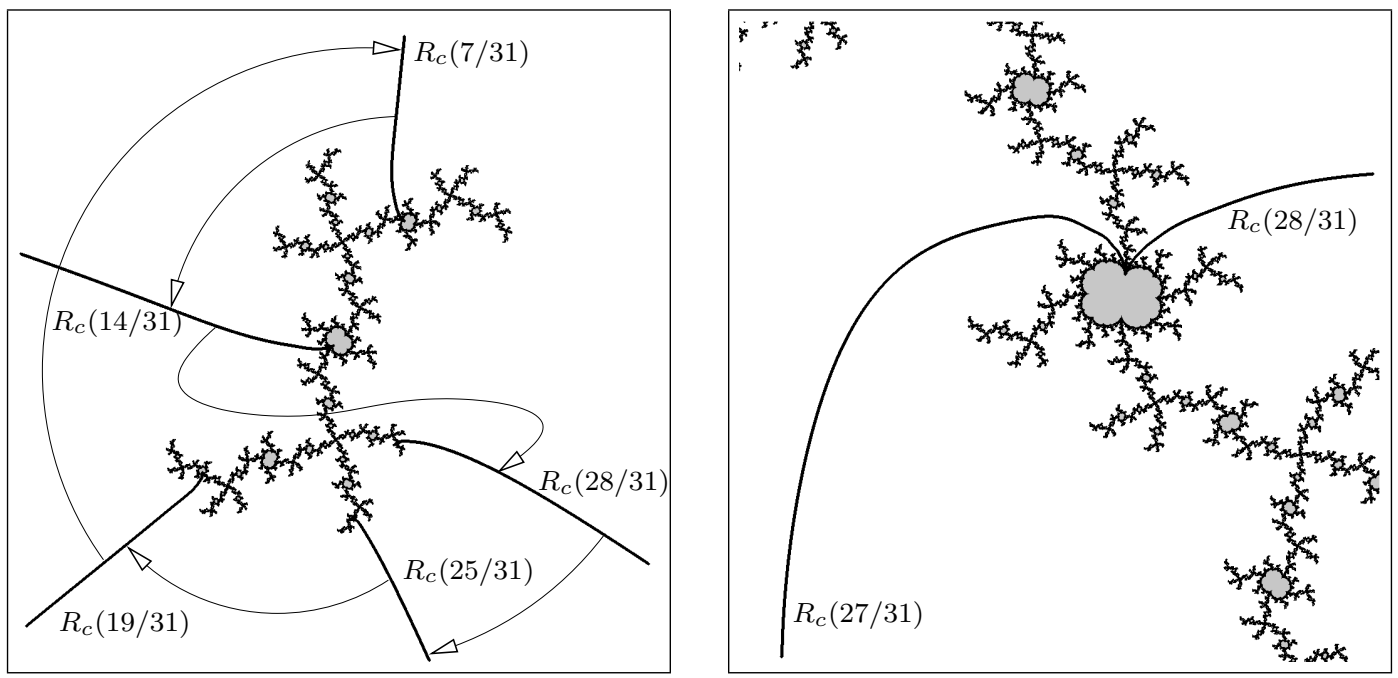

Figure 4. The filled-in Julia set $K_{c}$ and a cycle of dynamical rays for $c:=\gamma_{M}(28 / 31)$. The dynamical rays $R_{c}(28 / 31)$ and $R_{c}(27 / 31)$ both land at the same point.

at $c$. We say that $\theta$ and $\eta$ are companion angles. Both $\theta$ and $\eta$ are periodic under $\tau$ with the same period. The hyperbolic component is primitive if and only if the orbits of $\theta$ and $\eta$ under $\tau$ are distinct. Otherwise, the orbits are equal.

The dynamical rays $R_{c}(\theta)$ and $R_{c}(\eta)$ land at a common point $x_{1}:=\gamma_{c}(\theta)=\gamma_{c}(\eta)$. This point $x_{1}$ is the point of the parabolic cycle whose immediate basin contains the critical value $c$. The dynamical rays $R_{c}(\theta)$ and $R_{c}(\eta)$ are adjacent to the Fatou component containing $c$. The curve $R_{c}(\theta) \cup R_{c}(\eta) \cup\left\{x_{1}\right\}$ is a Jordan arc that cuts the plane in two connected components. One component, denoted $V_{0}$, contains the dynamical ray $R_{c}(0)$ and all the points of the parabolic cycle, except $x_{1}$. The other component, denoted $V_{1}$, contains the critical value $c$.

Since $V_{1}$ contains the critical value, its preimage $U_{\star}:=f_{c}^{-1}\left(V_{1}\right)$ is connected and contains the critical point 0 . It is bounded by the dynamical rays $R_{c}(\theta / 2), R_{c}(\eta / 2), R_{c}((\theta+1) / 2)$ and $R_{c}((\eta+1) / 2)$. Two of those dynamical rays land at the point $x_{0}$ of the parabolic cycle whose immediate basin contains the critical point 0 . The two other dynamical rays land at $-x_{0}$. Since $V_{0}$ does not contain the critical value, its preimage has two connected components. One component, denoted $U_{0}$, contains the dynamical ray $R_{c}(0)$. The other component is denoted $U_{1}$.

Lemma 4.3. Let $\theta \in \mathbb{T}$ be a periodic angle which is maximal in its orbit. Then,

- either $\theta=. \overline{11 \ldots 10}$

- or $\gamma_{M}(\theta)$ is the root of a primitive hyperbolic component.

Proof. If $\theta=0$, then $\gamma_{M}(0)=1 / 4$ is the root of a hyperbolic component. So, without loss of generality, we may assume that $\theta \neq 0$. Let $n \geq 2$ be the period of $\theta$ under $\tau$, let $\eta$ be the companion angle of $\theta$ and let $U_{0}$ and $U_{1}$ and $U_{\star}$ be defined as above.

Since $\theta$ is maximal in its orbit, $\tau^{\circ(n-1)}(\theta)=\theta / 2$ (see Lemma 4.2 ). So, $R_{c}(\theta / 2$ ) lands on $x_{0}$. One of the two rays $R_{c}(\eta / 2)$ and $R_{c}((\eta+1) / 2)$ lands on $x_{0}$. Since $U_{\star}$ is connected 


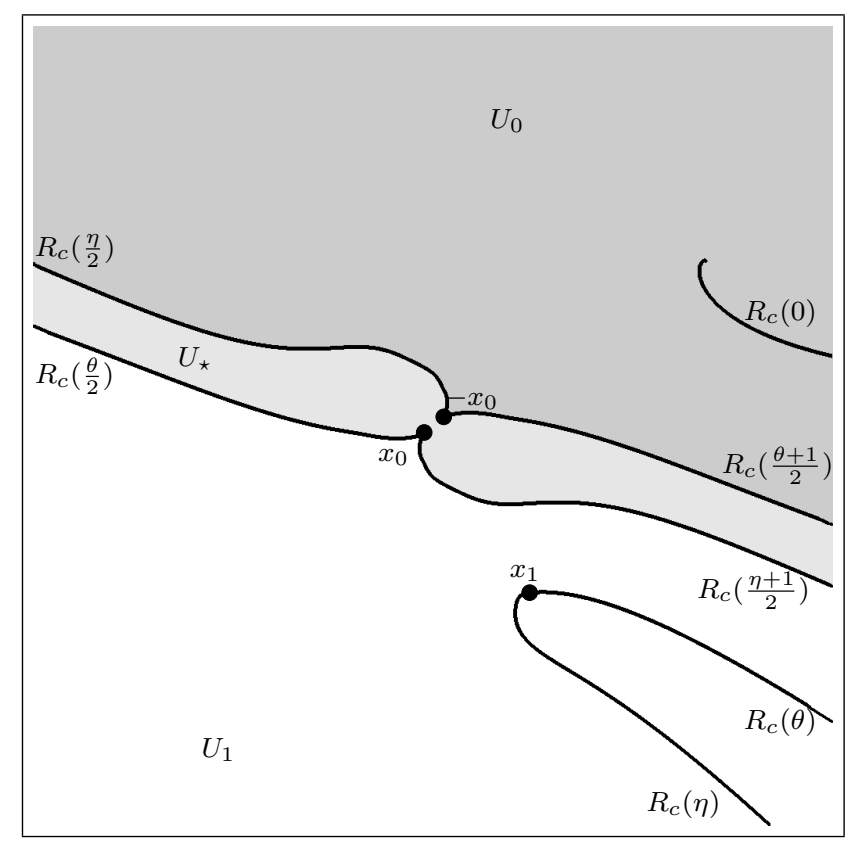

and contains dynamical rays with angles in between $\eta / 2$ and $\theta / 2$ and dynamical rays with angles in between $(\eta+1) / 2$ and $(\theta+1) / 2$, the ray landing on $x_{0}$ has to be $R_{c}((\eta+1) / 2)$. It follows that $(\eta+1) / 2$ is in the orbit of $\eta$ under $\tau$.

Since $\theta / 2<\theta<(\theta+1) / 2$ and since $R_{c}(\theta)$ avoids $U_{\star}$, we have $\theta \leq(\eta+1) / 2$. On the one hand, if $\theta<(\eta+1) / 2$, then the orbit of $\theta$ under $\tau$ does not contain $(\eta+1) / 2$ since otherwise $\theta$ would not be maximal in its orbit. In that case, the orbits of $\theta$ and $\eta$ are disjoint and $\gamma_{M}(\theta)$ is the root of a primitive hyperbolic component. On the other hand, if $\theta=(\eta+1) / 2$, then the rays $R_{c}(\theta)$ and $R_{c}((\eta+1) / 2)$ are equal. In that case, their landing point is the same, so $x_{0}=x_{1}=f_{c}\left(x_{0}\right)$ is a fixed point of $f_{c}$. The rays landing at this fixed point are permuted cyclically. The dynamical rays $R_{c}(\theta)$ and $R_{c}(\eta)$ are consecutive among the rays landing at $x_{0}, \eta<(\eta+1) / 2=\theta$ and $R_{c}(\theta)$ is mapped to $R_{c}(2 \theta)=R_{c}(\eta)$. It follows that each dynamical ray landing at $x_{0}$ is mapped to the one which is once further clockwise. Consequently, the kneading sequence of $\theta$ is $\overline{1 \ldots 1 \star}$ and according to Lemma 4.2 , the binary expansion of $\theta$ is.$\overline{1 \ldots 10}$.

4.3. Outside the Mandelbrot set. The projection $\pi_{c}: \bar{X}_{n} \rightarrow \mathbb{C}$ is a ramified covering. According to Proposition 3.1, the critical points are the points $(c, z) \in \bar{X}_{n}$ such that $f_{c}^{\circ n}(z)=z$ and $\left(f_{c}^{\circ n}\right)^{\prime}(z)=1$. So, the critical values are precisely the roots of the polynomial $\Delta_{n} \in \mathbb{Z}[c]$ which is the discriminant of $P_{n} \in \mathbf{Z}[z]$. Those critical values are contained in the Mandelbrot set since a parabolic cycle for $f_{c}$ attracts the critical point of $f_{c}$.

The open set

$$
W:=\mathbb{C}-\left(M \cup R_{M}(0)\right)
$$

is simply connected. It avoids the critical values of the ramified covering $\pi_{c}: \bar{X}_{n} \rightarrow \mathbb{C}$. Let $W_{n} \subset X_{n}$ be the preimage of $W$ by $\pi_{c}: \bar{X}_{n} \rightarrow \mathbb{C}$. It follows from the previous comment that $\pi_{c}: W_{n} \rightarrow W$ is a (unramified) cover, which is trivial since $W$ is simply connected: each connected component of $W_{n}$ maps isomorphically to $W$ by $\pi_{c}$. 

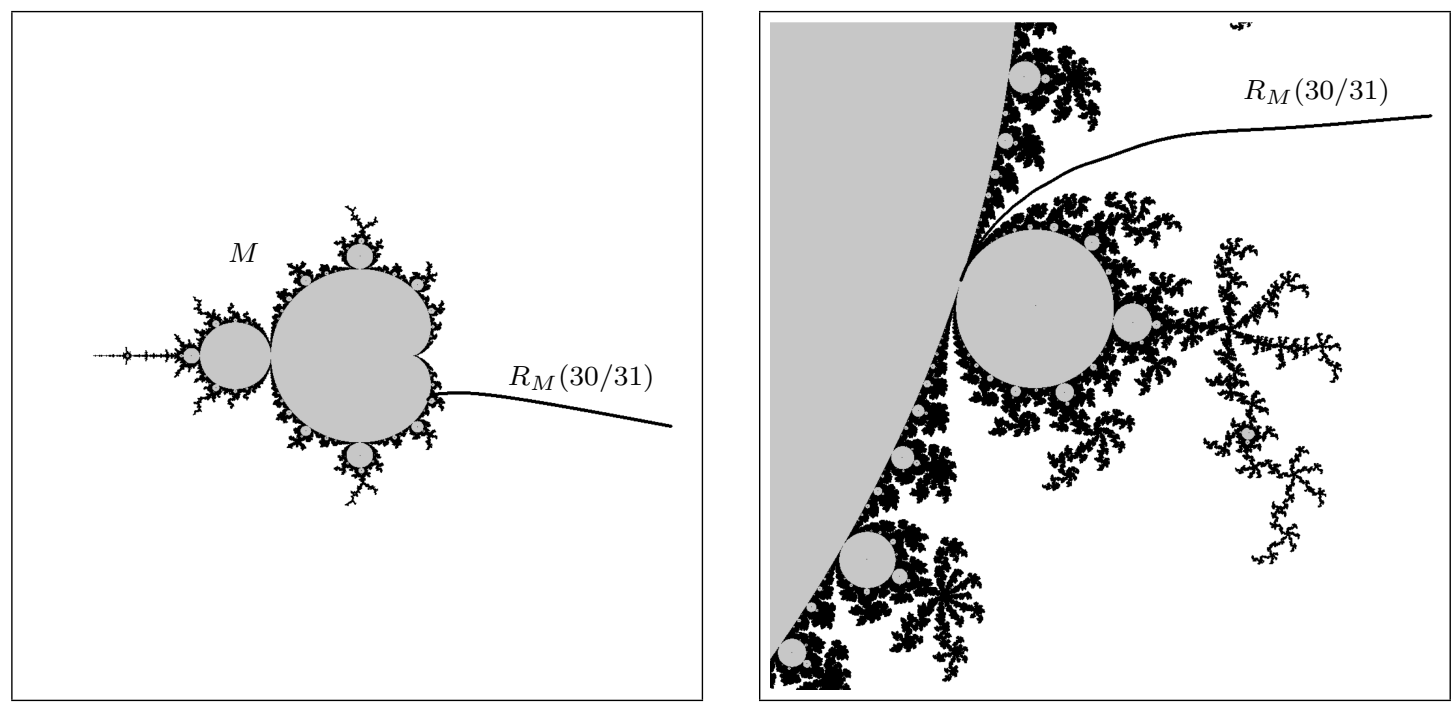

FiguRE 5. We have $30 / 31=. \overline{11110}$ and the parameter ray $R_{M}(30 / 31)$ lands on the boundary of the main cardioid.

Note that each connected component of $\bar{X}_{n}$ is unbounded (because $\bar{X}_{n}$ is an affine curve), and so, intersects $W_{n}$. Thus, in order to prove that $\bar{X}_{n}$ is connected, it is enough to prove that the closure $\bar{W}_{n}$ of $W_{n}$ in $\bar{X}_{n}$ is connected. We shall say that two components of $W_{n}$ are adjacent if they have a common boundary point in $\bar{X}_{n}$.

4.4. Labeling components of $W_{n}$. Here, we explain how the components of $W_{n}$ may be labelled dynamically.

A parameter $c \in W$ belongs to a parameter ray $R_{M}(\theta)$ with $\theta \neq 0$ not necessarily periodic. The dynamical rays $R_{c}(\theta / 2)$ and $R_{c}((\theta+1) / 2)$ bifurcate on the critical point. The Jordan curve $R_{c}(\theta / 2) \cup R_{c}((\theta+1) / 2) \cup\{0\}$ separates the complex plane in two connected components. We denote by $U_{0}(c)$ the component containing the dynamical ray $R_{c}(0)$ and by $U_{1}(c)$ the other component.

The orbit of a point $z \in K_{c}$ has an itinerary with respect to this partition. In other words, to each $z \in K_{c}$, we can associate a sequence $\iota_{c}(z) \in\{0,1\}{ }^{\mathbb{N}}$ whose $j$-th term is equal to 0 if $f_{c}^{\circ(j-1)}(z) \in U_{0}$ and is equal to 1 if $f_{c}^{\circ(j-1)}(z) \in U_{1}$. A point $z \in K_{c}$ is periodic for $f_{c}$ if and only if the itinerary $\iota_{c}(z)$ is periodic for the shift with the same period. The map $\iota_{c}: K_{c} \rightarrow\{0,1\}^{\mathbb{N}}$ is a bijection.

Let us define $\iota_{n}: W_{n} \rightarrow\{0,1\}^{\mathbb{N}}$ by

$$
\iota_{n}(c, z):=\iota_{c}(z)
$$

As $c$ varies in $W$, the periodic points of $f_{c}$, the dynamical ray $R_{c}(0)$ and the Jordan curve $R_{c}(\theta / 2) \cup R_{c}((\theta+1) / 2) \cup\{0\}$ move continuously. As a consequence, the map $\iota_{n}: W_{n} \rightarrow\{0,1\}^{\mathbb{N}}$ is locally constant, whence constant on each connected component of $W_{n}$. So, each connected component $V$ of $W_{n}$ may be labelled by the itinerary $\iota_{n}(V)$. Since $\iota_{c}: K_{c} \rightarrow\{0,1\}^{\mathbb{N}}$ is injective, distinct components have distinct labels. Since $\iota_{c}: K_{c} \rightarrow\{0,1\}^{\mathbb{N}}$ is surjective, each periodic itinerary of period $n$ is the label of a 


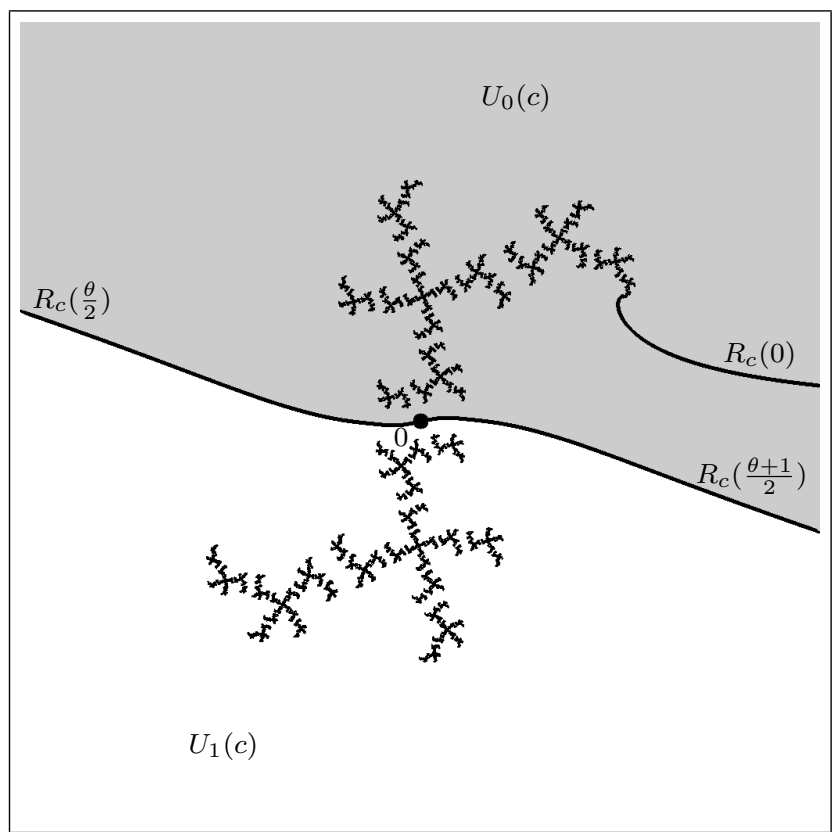

Figure 6 . The regions $U_{0}$ and $U_{2}$ for a parameter $c$ belonging to $R_{M}(28 / 31)$.

component of $W_{n}$. It follows that the number of connected components of $W_{n}$ is equal to the number of $n$-periodic sequences in $\{0,1\}^{\mathbb{N}}$.

4.5. Turning around critical points. We now exhibit connected components of $W_{n}$ which have common boundary points.

Proposition 4.4. Let $\overline{\varepsilon_{1} \ldots \varepsilon_{n-1 \star}}$ be the kneading sequence of an angle $\theta \in \mathbb{T}-\{0\}$ which is periodic of period $n$. If $\gamma_{M}(\theta)$ is the root of a primitive hyperbolic component and if one follows continuously the periodic points of period $n$ of $f_{c}$ as $c$ makes a small turn around $\gamma_{M}(\theta)$, then the periodic points with itineraries $\overline{\varepsilon_{1} \ldots \varepsilon_{n-1} 0}$ and $\overline{\varepsilon_{1} \ldots \varepsilon_{n-1} 1}$ get exchanged.

Proof. Set $c_{0}:=\gamma_{M}(\theta)$. Since $c_{0}$ is the root of a primitive hyperbolic component, the periodic point $x_{1}:=\gamma_{c_{0}}(\theta)$ has period $n$ and multiplier 1. According to Theorem 3.1 Case 2 (see also [DH, Exposé XIV, Proposition 3]), $X_{n}$ is smooth at $\left(c_{0}, x_{1}\right)$, the projection to the first coordinate has degree 2 and the projection to the second coordinate has degree 1. So, in a neighborhood of $\left(c_{0}, x_{1}\right)$ in $\mathbb{C}^{2}, X_{n}$ can be written as

$$
\left\{\left(c_{0}+\delta^{2}, x(\delta)\right),\left(c_{0}+\delta^{2}, x(-\delta)\right)\right\}
$$

where $x:(\mathbb{C}, 0) \rightarrow\left(\mathbb{C}, x_{1}\right)$ is a holomorphic germ with $x^{\prime}(0) \neq 0$. In particular, as $c$ moves away from $c_{0}$, the periodic point $x_{1}$ of $f_{c_{0}}$ splits into a pair of nearby periodic points $x\left( \pm \sqrt{c-c_{0}}\right)$ for $f_{c}$, that get exchanged when $c$ makes a small turn around $c_{0}$. So, it is enough to show that for $c \in \mathbb{C}-M$ close to $c_{0}$, those two periodic points have itineraries $\overline{\varepsilon_{1} \ldots \varepsilon_{n-1} 0}$ and $\overline{\varepsilon_{1} \ldots \varepsilon_{n-1} 1}$.

Let us denote by $V_{0}, V_{1}, U_{0}, U_{1}$ and $U_{\star}$ the open sets defined in Section 4.2. For $j \geq 0$, set $x_{j}:=f_{c_{0}}^{\circ j}\left(x_{0}\right)$ and observe that for $j \in[1, n-1]$, we have $x_{j} \in U_{\varepsilon_{j}}$. 
For $c \in R_{M}(\theta)$, consider the following compact subsets of the Riemann sphere $\mathbb{C} \cup\{\infty\}$ :

$$
R(c):=R_{c}(\theta) \cup\{c, \infty\} \quad \text { and } \quad S(c):=R_{c}(\theta / 2) \cup R_{c}((\theta+1) / 2) \cup\{0, \infty\} .
$$

Denote by $U_{0}(c)$ the component of $\mathbb{C}-S(c)$ containing $R_{c}(0)$ and by $U_{1}(c)$ the other component. From any sequence $c_{j} \in R_{M}(\theta)$ converging to $c_{0}$, we can extract a subsequence so that $R\left(c_{j}\right)$ and $S\left(c_{j}\right)$ converge respectively, for the Hausdorff topology on compact subsets of $\mathbb{C} \cup\{\infty\}$, to connected compact sets $R$ and $S$. Since $S(c)=f_{c}^{-1}(R(c))$, we have $S=f_{c_{0}}^{-1}(R)$. According to [PR, Sections 2 and 3], $R \cap\left(\mathbb{C}-K_{c_{0}}\right)=R_{c_{0}}(\theta)$, the intersection of $R$ with the boundary of $K_{c_{0}}$ is reduced to $\left\{x_{1}\right\}$, and the intersection of $R$ with the interior of $K_{c_{0}}$ is contained in the immediate basin of $x_{1}$, whence in $V_{1}$. It follows that as $c \in R_{M}(\theta)$ tends to $c_{0}$, any Hausdorff accumulation value of the family of compact sets $R(c)$ is contained in $\bar{V}_{1}$ and so, any accumulation value of the family of compact sets $S(c)$ is contained in $\bar{U}_{\star}$. In other words, any compact subset of $\mathbb{C}-\bar{U}_{\star}$ is contained in $\mathbb{C}-S(c)$ for $c \in R_{M}(\theta)$ close enough to $c_{0}$. More precisely, every compact subset of $U_{0}$ is contained in a connected compact set $L \subset U_{0}$ whose interior intersects $R_{c_{0}}(0)$; for $c \in R_{M}(\theta)$ close enough to $c_{0}, L$ intersects $R_{c}(0)$ and is contained in $\mathbb{C}-S(c)$, whence in $U_{0}(c)$. As a consequence, every compact subset of $U_{0}$ is contained in $U_{0}(c)$ for $c \in R_{M}(\theta)$ close enough to $c_{0}$. Similarly, every compact subset of $U_{1}$ is contained in $U_{1}(c)$ for $c \in R_{M}(\theta)$ close enough to $c_{0}$.

Fix $j \in[1, n-1]$ and let $D_{j}$ be a sufficiently small disk around $x_{j}$ so that

$$
\bar{D}_{j} \subset U_{\varepsilon_{j}} \subset \mathbb{C}-\bar{U}_{\star} .
$$

According to the previous discussion, if $c \in R_{M}(\theta)$ is close enough to $c_{0}$, we have

$$
f_{c}^{\circ(j-1)}\left(x\left( \pm \sqrt{c-c_{0}}\right)\right) \subset D_{j} \subset U_{\varepsilon_{j}}(c) .
$$

So, the itineraries of $x\left( \pm \sqrt{c-c_{0}}\right)$ are of the form $\overline{\varepsilon_{1} \ldots \varepsilon_{n-1} \varepsilon^{ \pm}}$with $\varepsilon^{ \pm} \in\{0,1\}$ and $\varepsilon^{+} \neq \varepsilon^{-}$(each itinerary corresponds to a unique point in $K_{c}$ ). The result follows.

Corollary 4.5. Let $\overline{\varepsilon_{1} \ldots \varepsilon_{n-1} \star}$ be the kneading sequence of an angle $\theta \in \mathbb{T}-\{0\}$ which is periodic of period $n$. If $\gamma_{M}(\theta)$ is the root of a primitive hyperbolic component, then, the components of $W_{n}$ with labels $\overline{\varepsilon_{1} \ldots \varepsilon_{n-1} 0}$ and $\overline{\varepsilon_{1} \ldots \varepsilon_{n-1} 1}$ are adjacent.

Proof. According to the previous proposition, the closures of those components both contain the point $\left(c_{0}, x_{1}\right)$ with $c_{0}:=\gamma_{M}(\theta)$ and $x_{1}:=\gamma_{c_{0}}(\theta)$.

Proposition 4.6. Let $\theta=1 /\left(2^{n}-1\right)=. \overline{1 \ldots 10}$ be periodic of period $n \geq 2$. If one follows continuously the periodic points of period $n$ of $f_{c}$ as $c$ makes a small turn around $\gamma_{M}(\theta)$, then the periodic points in the cycle of $\iota_{c}^{-1}(\overline{1 \ldots 10})$ get permuted cyclically.

Proof. Set $c_{0}:=\gamma_{M}(\theta)$. As mentioned earlier, all the dynamical rays $R_{c_{0}}\left(\tau^{\circ j}(\theta)\right)$ land on a common fixed point $x_{0}$. This fixed point is parabolic and each ray landing at $x_{0}$ is mapped to the one which is once further clockwise. It follows that the multiplier of $f_{c_{0}}$ at $x_{0}$ is $\omega:=\mathrm{e}^{-2 \pi \mathrm{i} / n}$.

According to Theorem 3.1 Case 3, $\bar{X}_{n}$ is smooth at $\left(c_{0}, x_{0}\right)$, the projection to the first coordinate has local degree $n$ and the projection to the second coordinate has local degree 1. It follows that in a neighborhood of $\left(c_{0}, x_{0}\right)$ in $\mathbb{C}^{2}, \bar{X}_{n}$ can be written as

$$
\left\{\left(c_{0}+\delta^{n}, x(\delta)\right),\left(c_{0}+\delta^{n}, x(\omega \delta)\right), \ldots,\left(c_{0}+\delta^{n}, x\left(\omega^{n-1} \delta\right)\right)\right\}
$$




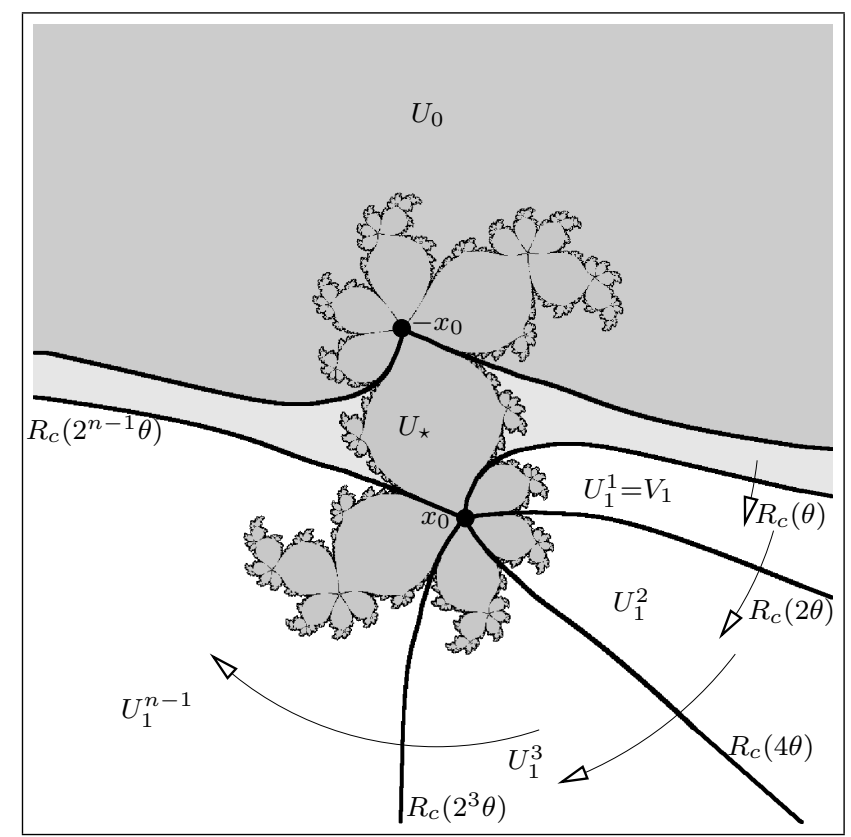

Figure 7 . For $c_{0}:=\gamma_{M}(. \overline{11110})$, the dynamical rays $R_{c_{0}}\left(\tau^{\circ j}(\theta)\right)$ land on a common fixed point $x_{0}$.

where $x:(\mathbb{C}, 0) \rightarrow\left(\mathbb{C}, x_{0}\right)$ is a holomorphic germ satisfying $x^{\prime}(0) \neq 0$. In addition,

$$
f_{c_{0}+\delta^{n}}(x(\delta))=x(\omega \delta) .
$$

So, for $c$ close to $c_{0}$, the set $\left.x\left\{\sqrt[n]{c-c_{0}}\right)\right\}$ is a cycle of period $n$ of $f_{c}$, and when $c$ makes a small turn around $c_{0}$, the periodic points in the cycle $\left.x\left\{\sqrt[n]{c-c_{0}}\right)\right\}$ get permuted cyclically. So, it is enough to show that for $c \in \mathbb{C}-M$ close enough to $c_{0}$, the point $\iota_{c}^{-1}(\overline{1 \ldots 10})$ belongs to $x\left\{\sqrt[n]{c-c_{0}}\right\}$.

Equivalently, we must show that there is a sequence $c_{j} \in \mathbb{C}-M$ converging to $c_{0}$, such that the sequence of periodic point $y_{j}=\iota_{c_{j}}^{-1}(\overline{1 \ldots 10})$ converges to $x_{0}$. Let $c_{j} \in R_{M}(\theta)$ converge to $c_{0}$. As in the previous proof, consider the following compact subsets of the Riemann sphere $\mathbb{C} \cup\{\infty\}$ :

$$
R\left(c_{j}\right):=R_{c_{j}}(\theta) \cup\left\{c_{j}, \infty\right\} \quad \text { and } \quad S\left(c_{j}\right):=R_{c_{j}}(\theta / 2) \cup R_{c_{j}}((\theta+1) / 2) \cup\{0, \infty\} .
$$

Denote by $U_{0}\left(c_{j}\right)$ the component of $\mathbb{C}-S\left(c_{j}\right)$ containing $R_{c_{j}}(0)$ and by $U_{1}\left(c_{j}\right)$ the other component. Without loss of generality, extracting a subsequence if necessary, we may assume that the sequence $y_{j}$ converges to a point $y$, and that the sequence $R\left(c_{j}\right)$ and $S\left(c_{j}\right)$ have Hausdorff limits $R$ and $S$. Passing to the limit on $f_{c_{j}}^{\circ n}\left(y_{j}\right)=y_{j}$, we see that $f_{c_{0}}^{\circ n}(y)=y$, and so, $y$ is periodic for $f_{c_{0}}$ with period dividing $n$. In particular, it is contained in the boundary of $K_{c_{0}}$. We must show that $y=\left\{x_{0}\right\}$.

It follows from $\left[\mathrm{PR}\right.$, Sections 2 and 3] that $R \cap\left(\mathbb{C}-K_{c_{0}}\right)=R_{c_{0}}(\theta)$, the intersection of $R$ with the boundary of $K_{c_{0}}$ is reduced to $\left\{x_{0}\right\}$ and the intersection of $R$ with the interior of $K_{c_{0}}$ is contained in the immediate basin of $x_{0}$. We cannot quite conclude that $L$ is contained in $\bar{U}_{\star}$, but rather that it is contained in $\bar{U}_{\star} \cup \stackrel{\circ}{K}_{c_{0}}$. As in the previous proof, it follows that the Hausdorff limit of $\bar{U}_{1}\left(c_{j}\right)$ is contained in $\bar{U}_{\star} \cup U_{1} \cup \stackrel{\circ}{K_{c_{0}}}$. Since $\iota_{c_{j}}\left(y_{j}\right)=\overline{1 \ldots 10}$, we know that $y_{j}, f_{c_{j}}\left(y_{j}\right), \ldots, f_{c_{j}}^{\circ(n-2)}\left(y_{j}\right)$ belong to $U_{1}\left(c_{j}\right)$. So the points 
$y, f_{c_{0}}(y), \ldots, f_{c_{0}}^{\circ(n-2)}(y)$ belong to $\bar{U}_{\star} \cup U_{1} \cup \stackrel{\circ}{K}_{c_{0}}$. Since $y$ is in the boundary of $K_{c_{0}}$, we deduce that $y, f_{c_{0}}(y), \ldots, f_{c_{0}}^{\circ(n-2)}(y)$ belong to $\bar{U}_{\star} \cup U_{1}$.

The dynamical rays landing at $x_{0}$ divide $U_{1}$ in $n-1$ connected components $U_{1}^{j}$ labelled clockwise so that

$$
V_{1}=U_{1}^{1} \stackrel{f_{c_{0}}}{\longrightarrow} U_{1}^{2} \stackrel{f_{c_{0}}}{\longrightarrow} \cdots \stackrel{f_{c_{0}}}{\longrightarrow} U_{1}^{n-1} \stackrel{f_{c_{0}}}{\longrightarrow} \mathbb{C}-\bar{U}_{1} .
$$

The component $U_{\star}$ maps with degree 2 to $U_{1}^{1}=V_{1}$ (see Figure 7 ).

Now, we claim that the orbit of $y$ intersects $\bar{U}_{\star}$. Indeed, either $y$ itself is in $\bar{U}_{\star}$, or $y$ is in $U_{1}^{j}$ for some $j \geq 1$. Then, $f_{c_{0}}^{\circ(n-j)}(y) \in \mathbb{C}-\bar{U}_{1}$. Since it cannot be in $U_{0}$, it belongs to $\bar{U}_{\star}$.

The map $f_{c_{0}}^{\circ n}: U_{\star} \rightarrow \mathbb{C}-\bar{U}_{1}$ is a proper map of degree 2 and $U_{\star} \subset \mathbb{C}-\bar{U}_{1}$. Note that $x_{0} \in \bar{U}_{\star}$ is a multiple fixed point of $f_{c_{0}}^{\circ n}$ and that there is an attracting petal contained in $U_{\star}$. It follows from a version of the Lefschetz fixed point formula (see [GM, Lemma 3.7]) that $x_{0}$ is the only fixed point of $f_{c_{0}}^{\circ n}$ contained in $\bar{U}_{\star}$.

As a consequence, the orbit of the periodic point $y$ contains $x_{0}$, and since $x_{0}$ is a fixed point, we have $y=x_{0}$ as required.

Corollary 4.7. The components of $W_{n}$ whose labels contain a single 0 are adjacent.

Proof. Let $\theta:=\overline{1 \ldots 10}$ and $\left(c_{0}, x_{0}\right):=\left(\gamma_{M}(\theta), \gamma_{c_{0}}(\theta)\right)$. By the above proposition every component of $W_{n}$ whose label is a shift of $\overline{1 \ldots 10}$ contains $\left(c_{0}, x_{0}\right)$ in its boundary. But every periodic label of period $n$ containing a single 0 is indeed a shift of $\overline{1 \ldots 10}$.

4.6. Proof of Theorem 4.1. We will finally deduce that $\bar{W}_{n}$ is connected. According to Corollary 4.7, components of $W_{n}$ whose label contain a single 0 have a common boundary point. So, it is enough to show that component of $W_{n}$ whose label has at least two 0 has a common boundary point with a component of $W_{n}$ whose label has one less 0 .

The map $F: \mathbb{C}^{2} \rightarrow \mathbb{C}^{2}$ defined by

$$
F(c, z):=\left(c, f_{c}(z)\right)
$$

restricts to an isomorphism $F: X_{n} \rightarrow X_{n}$. It permutes the components of $W_{n}$ as follows: the label of $F(C)$ is the shift of the label of $C$. In addition, two components $C_{1}$ and $C_{2}$ of $W_{n}$ are adjacent if and only if $F\left(C_{1}\right)$ and $F\left(C_{2}\right)$ are adjacent.

Let $C$ be a connected component of $W_{n}$ whose label $\iota$ contains at least two 0 . Let $\overline{\varepsilon_{1} \ldots \varepsilon_{n}}=\sigma^{\circ k}(\iota)$ be maximal (in the lexigographic order) among the iterated shifts of $\iota$. Then, the angle $\theta:=. \overline{\varepsilon_{1} \ldots \varepsilon_{n}}$ is maximal in its orbit. According to Lemma 4.2, $\varepsilon_{n}=0$ and the kneading sequence $\nu(\theta)$ is $\overline{\varepsilon_{1} \ldots \varepsilon_{n-1} \star}$. According to Lemma 4.3. $\gamma_{M}(\theta)$ is the root of a primitive hyperbolic component. According to Corollary 4.5 , the component $F^{\circ k}(C)$ which is labeled $\overline{\varepsilon_{1} \ldots \varepsilon_{n-1} 0}$ is adjacent to the component $C^{\prime}$ of $W_{n}$ which is labeled $\overline{\varepsilon_{1} \ldots \varepsilon_{n-1} 1}$. Then, $F^{\circ(n-k)}\left(C^{\prime}\right)$ is a component of $W_{n}$ adjacent to $F^{\circ(n-k)}\left(F^{\circ k}(C)\right)=C$, and its label contains one less 0 than the label of $C$.

This completes the proof of Theorem 4.1. 


\section{REFERENCES}

[B] T. Bousch, Sur quelques problèmes de dynamique holomorphe, Ph.D. thesis, Université de ParisSud, Orsay, 1992.

[BKM] A. Bonifant, J. Kiwi \& J. Milnor, Cubic polynomial maps with periodic critical orbit. II. Escape regions, Conform. Geom. Dyn. 14 (2010), 68-112.

[DH] A. Douady and J.H. Hubbard, Etude dynamique des polynômes complexes (Deuxième partie), 1985.

[E] A.L. Epstein, Transversality Principles in Holomorphic Dynamics, Preprint.

[GM] L.R. Goldberg \& J. Milnor, Fixed points of polynomial maps. Part II. Fixed point portraits. Ann. Sci. Éc. Norm. Supér., IV. Sér. 26, No. 1, 51-98 (1993).

[LS] E. LAU \& D. SchleICher, Internal addresses in the Mandelbrot set and irreducibility of polynomials, Stony Brook Preprint 19, 1994.

[L] G. Levin, On explicit connections between dynamical and parameter spaces, Journal d'Analyse Mathematique, 91( 2003), 297-327.

[Mi1] J. Milnor, Geometry and dynamics of quadratic rational maps, Experiment. Math., 2(1):37-83, 1993. With an appendix by the author and Tan Lei.

[Mi2] J. Milnor, Tsujii's monotonicity proof for real quadratic maps, Preprint http://www.math. sunysb.edu/ jack/PREPRINTS/tsujii.ps.

[Mi3] J. Milnor, Cubic polynomial maps with periodic critical orbit. I, in Complex dynamics, Families and friends, ed. D. Schleicher, A K Peters, Wellesley, MA (2009), 333-411.

[Mo] P. Morton, On certain algebraic curves related to polynomial maps, Compositio Math. 103 (1996), no. 3, 319-350.

[PR] C. L. Petersen \& G. Ryd, Convergence of rational rays in parameter spaces, in 'The Mandelbrotset, Theme and Variations. Edited by Tan Lei, London Mathematical Society, Lecture Note Series 274. Cambridge University Press 2000.

[Sc] D. SchleIcher, Internal addresses of the Mandelbrot set and Galois groups of polynomials, arXiv:math/9411238v2, Feb. 2008.

[Si] J.H. Silverman, The arithmetic of dynamical systems, Graduate Texts in Math. 241, Springer, New York, 2007. 